Comparison of 3D flux-driven scrape-off layer turbulence simulations with gas-puff imaging of Alcator C-Mod inner-wall limited discharges

This content has been downloaded from IOPscience. Please scroll down to see the full text.

View the table of contents for this issue, or go to the journal homepage for more

Download details:

This content was downloaded by: riccipaolo

IP Address: 128.179.252.199

This content was downloaded on 02/05/2015 at 03:54

Please note that terms and conditions apply. 


\title{
Comparison of 3D flux-driven scrape-off layer turbulence simulations with gas-puff imaging of Alcator C-Mod inner-wall limited discharges
}

\author{
F D Halpern ${ }^{1}$, J L Terry², S J Zweben ${ }^{3}$, B LaBombard ${ }^{2}$, M Podesta ${ }^{3}$ and \\ P Ricci ${ }^{1}$ \\ ${ }^{1}$ École Polytechnique Fédérale de Lausanne (EPFL), Centre de Recherches en Physique des Plasmas \\ (CRPP), CH-1015 Lausanne, Switzerland \\ 2 Massachusetts Institute of Technology, 77 Massachusetts Avenue, Cambridge, MA 02139, USA \\ 3 Princeton Plasma Physics Laboratory, Princeton, NJ 08540, USA \\ E-mail: federico.halpern@epfl.ch
}

Received 9 October 2014, revised 16 December 2014

Accepted for publication 2 January 2015

Published 15 April 2015

\begin{abstract}
We carry out a quantitative comparison between gas-puff imaging (GPI) turbulence measurements in Alcator C-Mod inner-wall limited discharges (Zweben et al 2009 Phys. Plasmas 18 082505) and 3D flux-driven drift-reduced Braginskii turbulence simulations of scrape-off layer dynamics. The comparison is carried out for a series of inner-wall limited discharges where the magnetic field and the density are varied. The comparison between GPI data and non-linear simulations yields overall good agreement for several observables, such as the $D_{\alpha}$ emission levels and intermittency, the radial and poloidal correlation lengths and propagation velocities, and the power and frequency spectral density.
\end{abstract}

Keywords: turbulence, gas-puff imaging, flux-driven, scrape-off layer

(Some figures may appear in colour only in the online journal)

\section{Introduction}

Understanding tokamak scrape-off layer (SOL) dynamics is an important scientific challenge to address as we approach the era of burning plasma experiments. The SOL plays a crucial role in tokamak discharges, for instance, by controlling the impurity influx into the core plasma, the recycling level, and the heat exhaust. The SOL width, in particular, determines the power deposition onto plasma facing components.

The relatively low SOL temperatures $\lesssim 100 \mathrm{eV}$, and its proximity to the vacuum vessel allow very good diagnostic access, for instance, for Langmuir probes and for gas-puff imaging (GPI) systems. Experimental measurements in many tokamaks have shown that the SOL dynamics are characterized by strongly non-linear behavior [1]. Fluctuations have a very large relative amplitude, $\delta n / n \sim 1$, and their radial and poloidal wavelengths are of the same order as the radial extension of the SOL (we denote the fluctuating component as $\delta n / n=\left(n-\langle n\rangle_{t}\right) /\langle n\rangle_{t}$, with \langle\rangle$_{t}$ indicating a time average $)$. The turbulent correlation times, of a few tens of microseconds, are also comparable with the parallel transit time $\sim R / c_{s}$, that is, the plasma permanence time in the SOL ( $R$ is the tokamak major radius and $c_{s}=\sqrt{\left(T_{e}+T_{i}\right) / m_{i}}$ is the sound speed). Finally, it is believed that a large fraction of the radial transport is due to non-linear intermittent transport events denominated as blobs [2]. As a result, SOL turbulence must be approached through a global and flux driven description, resolving both micro (up to $\left.\rho_{s}\right)$ and macroscopic $\left(L_{p}\right)$ length scales, and without formally separating fluctuations from background profiles.

In the past decade, computational advances have increased our understanding of SOL dynamics and helped interpret experimental results. The fluctuation levels and intermittency 
found in the tokamak SOL were handily described by $2 \mathrm{D}$ flux-driven turbulence codes such as TOKAM2D, ESEL, and SOLT [3-6]. These codes included the physics of blob filaments and of curvature driven modes in the absence of parallel dynamics. Newer, 3D models also included some potentially important effects, such as drift-wave turbulence and magnetic fluctuations. Advanced, electromagnetic $\delta f$ gyrofluid models, such as GEMR [7], were also used to describe global SOL dynamics [8, 9].

The increase of computational power in recent years has allowed 3D global, flux-driven simulations of the tokamak SOL using GBS [10], a numerical implementation of the two fluid drift-reduced Braginskii model [11]. In effect, the saturation mechanism and the non-linear turbulent regimes in limiter configuration were identified $[12,13]$. The size scaling of the SOL width for inner-wall limited plasmas was found analytically and its scaling was confirmed using simulations that varied the normalized plasma size [14]. This effort has resulted in a quantitative comparison between a simple analytical scaling and the pressure decay length measured with Langmuir probes in several tokamaks [15].

Following this theory-experiment comparison between the pressure decay lengths, the goal of the present work is to carry out a quantitative comparison between GPI measurements of SOL turbulent dynamics, performed in Alcator C-Mod [9, 16], and flux-driven turbulence simulations carried out with GBS. The comparison includes a set of 21 inner-wall limited discharges with $B=2.7 \mathrm{~T}$ and $B=3.8 \mathrm{~T}$, which are within the reach of present computational capabilities of GBS. In order to facilitate the comparison, a simple synthetic diagnostic for $D_{\alpha}$ emission was applied to the GBS data. The root-meansquare fluctuation levels, intermittency, correlation lengths, the auto-correlation time, turbulent propagation velocities, and frequency and wave number spectral powers were all compared against GPI measurements.

As opposed to a previous paper involving the same discharges [9], where the turbulence was calculated using fixed profiles taken from the experiment, here we aim to also produce reasonable time averaged profiles-which in turn drive the turbulence. The only 'adjustable parameters' in the simulations are source terms, which are adjusted to match the reference temperature and density at the last closed flux serfice (LCFS) obtained from the experiment. The profile gradients are a self-consistent result of the simulated SOL dynamics. For this reason, the present comparison is a both a stringent and challenging test. GBS must first, through the interaction of turbulent and background length scales, produce reasonable time averaged profiles, which then must give rise to turbulent modes comparable to those observed in the experiment. Remarkably, the simulations results yield very good agreement with the GPI measurements.

This paper is organized as follows. First, in section 2, we describe the experimental data set used for the comparison. Section 3 gives an introduction to computational model and setup used to simulate the Alcator C-Mod SOL. In section 4 we compare the results of the simulations against the GPI measurements. Some final remarks summarizing the main results of the comparison are found in section 5 .

\section{Alcator C-Mod discharge parameters and diagnostics}

The present comparison involves inner-wall limited, near circular $(\kappa=1.2)$, deuterium Ohmic plasmas carried out in Alcator C-Mod and described in [9]. For completeness, we briefly recall the main properties of the discharges used. The experimental set-up of Alcator C-Mod at the time the discharges took place is described in [16]. The experiments consisted in a series of discharges at low and intermediate magnetic field $(B=2.7 \mathrm{~T}$, and $3.8 \mathrm{~T})$ with $q_{a} \approx 3$. A density scan was carried out at each value of the magnetic field, although the line averaged density $\left\langle n_{e}\right\rangle$ stayed well below the Greenwald density limit. The relative simplicity of the geometry of these plasmas combined with the GPI and probe diagnostics makes them ideal for a comparison with a code such as GBS.

The diagnostics used to study SOL turbulence and profiles were the Alcator C-Mod reciprocating Langmuir probe and the gas puff imaging (GPI) system [17]. Measurements of the plasma profiles, as well as turbulent quantities such as fluctuation amplitude and turbulent velocities were carried out around the low-field-side mid plane region of C-Mod. In order to improve diagnostic access to the SOL, the plasmas were shaped to maximize the outer mid plane gap. The position of the LCFS, obtained from equilibrium reconstruction, was $R \approx 86.7 \mathrm{~cm}$ while the wall was at $R=90.5 \mathrm{~cm}$, which gives a gap of $\sim 4 \mathrm{~cm}$. Plasma conditions were constant for roughly between $t=0.7-1.3 \mathrm{~s}$, with the time of interest for the comparisons being $t \approx 1 \mathrm{~s}$, which is near the peak of the GPI diagnostic gas puff. The line averaged density increased by $5-30 \%$ with the gas puff-however, no significant change in the turbulent quantities was observed over the length of the GPI measurements.

SOL turbulent quantities were evaluated using fluctuations in $D_{\alpha}$ light emitted by a neutral deuterium gas puff near the LFS mid plane. The detection system used for these discharges consists of a series of discrete fast photodiodes coupled to fiber views that are arranged into a vertical array and a horizontal array at about $R=89 \mathrm{~cm}$. Each detector has a viewing diameter of $3 \mathrm{~mm}$. The signals were digitized at $1 \mathrm{MHz}$ and had an effective bandwidth of $0.4 \mathrm{MHz}$. The positions of the views with respect to the LCFS are obtained from equilibrium reconstruction. DEGAS-2 calculations including neutral transport and atomic physics processes were carried out in order to aid the interpretation of the GPI measurements [18]. DEGAS-2 estimated the toroidal extent of the $D_{\alpha}$ emission to be roughly $6 \mathrm{~cm}$, which together with a $8^{\circ}$ angle between the GPI viewing chords and the magnetic field lines gives some additional smoothing ( $\sim 8 \mathrm{~mm}$ in the poloidal direction).

In the present study we consider several discharges with $B=2.7 \mathrm{~T}$ and $B=3.8 \mathrm{~T}$ at different density. It has been verified that the discharges selected are not affected by MARFEs, which would affect the SOL dynamics. To facilitate the comparison and reduce the number of simulations to carry out, we group the $B=2.7$ and $B=3.8 \mathrm{~T}$ discharges into 'low density' and 'high density' scenarios (table 1). For the low $B$ data set, in particular, several discharges exist and small variations of the plasma position allow, in effect, to obtain radial profiles 
Table 1. Alcator C-Mod parameters used to initialize the GBS simulations.

\begin{tabular}{llllll}
\hline$B_{0}[T]$ & $\bar{n}\left[10^{19} \mathrm{~m}^{-3}\right]$ & $\bar{T}_{e}[\mathrm{eV}]$ & $\nu$ & $\rho_{\star}^{-1}$ & $L_{y}$ \\
\hline 2.7 & 1.66 (low) & 21.1 & 0.0125 & 1572 & 3184 \\
2.7 & 3.73 (high) & 29.4 & 0.0146 & 1333 & 2700 \\
3.8 & 4.75 (low) & 36.8 & 0.0119 & 1678 & 3399 \\
3.8 & 6.67 (high) & 26.6 & 0.0318 & 1971 & 3991 \\
\hline
\end{tabular}

of the quantities measured using the radial array sensor. The poloidal correlation lengths and velocity, on the other hand, are only known at the location of the vertical GPI sensor array.

\section{Simulation model and setup}

The drift-reduced Braginskii equations used in the C-Mod simulations are presented in detail in $[10,11]$. They are derived starting from the Braginskii two fluid equations [19] by imposing the orderings $\mathrm{d} / \mathrm{d} t \ll \omega_{c i}, k_{\perp} \gg k_{\|}$. We have employed an electrostatic model, since $\beta_{e} \sim 10^{-5}$ and we expect $\alpha=q^{2} \beta_{e} R / L_{p} \ll 1$ for these simulations. A model with constant ion temperature is used. Finite ion temperature effects are considered in [20], the principal result being that curvature driven modes (i.e. resistive ballooning modes) are slightly enhanced with respect to the cold ion model. The drift-reduced equations, in normalized units, read as follows:

$$
\begin{aligned}
\partial_{t} n=- & \frac{\rho_{\star}^{-1}}{B}[\phi, n]-\nabla_{\|}\left(n v_{\| e}\right)+S_{n}+\frac{2}{B}\left[\hat{C}\left(p_{e}\right)-n \hat{C}(\phi)\right] \\
& +D_{n} \nabla_{\perp}^{2} n \\
\partial_{t} \omega=- & \frac{\rho_{\star}^{-1}}{B}[\phi, \omega]-v_{\| i} \nabla_{\|} \omega+\frac{\hat{C}\left(G_{i}\right)}{3 n}+\frac{2 B}{n} \hat{C}\left(p_{e}\right) \\
& +\frac{B^{2}}{n} \nabla_{\|} j_{\|}+D_{\omega} \nabla_{\perp}^{2} \omega \\
\partial_{t} v_{\| e}=- & \frac{\rho_{\star}^{-1}}{B}\left[\phi, v_{\| e}\right]-v_{\| e} \nabla_{\|} v_{\| e} \\
& +\frac{m_{i}}{m_{e}}\left(\nu \frac{j_{\|}}{n}+\nabla_{\|} \phi-\frac{1}{n} \nabla_{\|} p_{e}-0.71 \nabla_{\|} T_{e}\right) \\
& -\frac{2 B m_{i}}{3 n m_{e}} \nabla_{\|} G_{e}+D_{v_{\| e}} \nabla_{\perp}^{2} v_{\| e} \\
& +\frac{2}{3} T_{e}\left(\frac{0.71}{n} \nabla_{\|} j_{\|}-\nabla_{\|} v_{\| e}\right)+D_{T_{e}} \nabla_{\perp}^{2} T_{e}, \\
\partial_{t} v_{\| i}=- & \frac{\rho_{\star}^{-1}}{B}\left[\phi, v_{\| i}\right]-v_{\| i} \nabla_{\|} v_{\| i}-\frac{2 B}{3 n} \nabla_{\|} G_{i}-\frac{1}{n} \nabla_{\|} p_{e} \\
& +D_{v_{\| i}} \nabla_{\perp}^{2} v_{\| i} \\
& \frac{\rho_{\star}^{-1}}{B}\left[\phi, T_{e}\right]-v_{\| e} \nabla_{\|} T_{e}+S_{T_{e}} \\
+ & \left.T_{e}\left(T_{e}\right)+\frac{T_{e}}{n} \hat{C}(n)-\hat{C}(\phi)\right] \\
\partial_{t} &
\end{aligned}
$$

where $\omega=\nabla_{\perp}^{2} \phi$ is the vorticity and equation (2) has been simplified using the Boussinesq approximation $\nabla \cdot\left(n d_{t} \nabla_{\perp} \phi\right) \approx n d_{t} \nabla_{\perp}^{2} \phi$. The parallel current is given by $j_{\|}=n\left(v_{\| i}-v_{\| e}\right)$.

The following normalizations are used in the drift-reduced equations: $t=\tilde{t} /\left(\bar{R} / \bar{c}_{s}\right), \quad \nabla_{\perp}=\bar{\rho}_{s} \widetilde{\nabla}_{\perp}, \quad \nabla_{\|}=\bar{R} \widetilde{\nabla}_{\|}, \quad v_{\|}=\widetilde{v}_{\|} / \bar{c}_{s}$, $B=\widetilde{B} / \bar{B}, n=\tilde{n} / \bar{n}, T_{e}=\widetilde{T}_{e} / \bar{T}_{e}$, and $\phi=e \widetilde{\phi} / \bar{T}_{e}$. Here, the tildes denote quantities in MKS physical units, and the bars denote reference quantities defined in terms of reference values (e.g. $\bar{c}_{s}=\sqrt{\bar{T}_{e}(1+\tau) / m_{i}}$, with $\left.\tau=\bar{T}_{i} / \bar{T}_{e}\right)$. The ion temperature enters only through the reference value of the sound speed. The reference density $\bar{n}$ and temperature $\bar{T}_{e}$ and $\bar{T}_{i}$ are taken at the LCFS, while $\bar{R}$ and $\bar{B}$ are defined at the magnetic axis. All variables are expressed in their dimensionless form unless specified otherwise.

The dimensionless parameters used are as follows: the adimensional Spitzer resistivity is $\nu=e^{2} \bar{n} \bar{R} /\left(m_{i} \sigma_{\|} \bar{c}_{s}\right)$, with $\sigma_{\|}$ begin the standard Spitzer conductivity defined at the LCFS using the reference $n$ and $T_{e}$; the plasma size is given by the inverse of $\rho_{\star}=\bar{\rho}_{s} / \bar{R}=\sqrt{\bar{T}_{e}(1+\tau) / m_{i}} /\left(e \bar{B} \bar{R} / m_{i}\right)$, and the poloidal length of the system is $L_{y}=2 \pi \bar{a} / \bar{\rho}_{s}, \bar{a}$ is the minor radius.

Plasma outflow from the closed flux surface region is mimicked using density and temperature sources, respectively, $S_{n}$ and $S_{T_{e}}$. The $G_{e}$ and $G_{i}$ terms represent the gyroviscous part of the pressure tensor (see [10]). Small perpendicular diffusion terms of the form $D_{f} \nabla_{\perp}^{2} f$ are added to avoid the pile-up of aliased modes at the Nyquist resolution of the numerical grid. The numerical value of the diffusion coefficients is $D_{f}=2$. We have verified, a posteriori, that these terms drive less than $1 \%$ of the total radial flux in the non-linear stage. In addition, $[f, g]=\mathbf{B} \cdot(\nabla f \times \nabla g) / B$ is the Poisson bracket, while $\hat{C}(f)=(B / 2)\left[\nabla \times\left(\mathbf{B} / B^{2}\right)\right] \cdot \nabla f$ is the curvature operator.

We consider a SOL model in circular geometry with a toroidal limiter set at the high-field side equatorial mid plane. The coordinate system used is $(\theta, r, \varphi)$, right-handed$r$ is the radial coordinate, with $r=0$ set at the last closed flux-surface, $\theta$ is the poloidal angle, and $\varphi$ is the toroidal angle. Under these assumptions, the curvature operator reduces to $\hat{C}(f)=(\sin \theta) \partial_{r} f+(\cos \theta+\hat{s} \theta \sin \theta) \partial_{\theta} f$ and the Poisson bracket is defined as $[f, g]=a^{-1}\left(\partial_{\theta} f \partial_{r} g-\partial_{r} f \partial_{\theta} g\right)$ $\left(\hat{s}=(a+r) q^{\prime} / q\right.$ is the magnetic shear).

The plasma interfaces with the vacuum vessel through a magnetized pre-sheath where the fluid drift approximation breaks down, as ions and electrons are accelerated by a strong electric field. The validity of the drift-reduced model, therefore, formally extends until the magnetic pre-sheath entrance, where we apply a set of generalized Bohm-Chodura boundary conditions developed from a kinetic model [21]

Since in a previous paper [9] a similar comparison between Alcator C-Mod data and non-linear turbulence simulations was carried out using the GEMR code [22, 23], it is worth describing some of the differences between the GEMR and GBS approaches. The goal of the previous study [9] (using GEMR) was to address the properties of C-Mod SOL turbulence within a small region of the C-Mod SOL for a given set of static parameters (i.e. pressure gradient length, collisionality, plasma $\beta$, etc). GEMR is an electromagnetic gyrofluid 
code, which is valid at arbitrary collisionality, with radially varying profiles in a global domain. The equations used by GEMR are derived by taking fluid moments of the $\delta f$ gyrokinetic equations, which results in a self-consistent model. According to the gyrokinetic ordering, use of $\delta f$ gyrokinetics (and derived models) requires small amplitude fluctuations. The simulations presented in [9] used Bohm sheath boundary conditions as implemented in [8]. Both GEMR and GBS neglect compressional Alfvén dynamics and trapped particles.

GBS, on the other hand, is a global and flux-driven code based on the drift-reduced Braginskii equations [11], which are valid only at high collisionality where the distribution function stays close to Maxwellian. There are several differences between the two approaches. In GBS simulations, the plasma profiles are built during the simulation through the use of particle and heat sources, which mimic the plasma outflow from the closed field line region. Rather than assuming a background scale length, the temperature and density profiles are evolved self-consistently with the evolution of turbulent eddies. In fact, the time averaged SOL width in GBS results from a power balance between particle and heat injection, turbulent transport, and parallel losses at the limiters. Plasma outflow into a toroidal limiter, which is placed on the high-field-side for this work, is modeled using first-principles sheath boundary conditions [21]. Thus, with GBS we concentrate on describing a turbulent plasma without placing any assumptions on the amplitude of the modes or the length scales involved.

It is worth mentioning that existing simulations using the two approaches yield some similar insights on SOL dynamics. Electromagnetic GBS simulations typically report resistive ballooning mode turbulence during the non-linear quasi-steady-state, with electromagnetic effects not playing a major role even at realistic parameters and machine size [14, 24]. The saturation level and time averaged profiles are well described by the gradient removal model [12]. Similarly, Ribeiro and Scott [8] carried out SOL turbulence simulations with a toroidal limiter, finding the prevalence of ballooning-like turbulence over drift-waves. They also proposed the pressure non-linearity as a saturation mechanism.

However, since GBS is a flux driven code, the background quantities adjust themselves with the turbulent fluxes, resulting in self-consistent profiles. This has allowed a quantitative verification of the saturation mechanism [14], and the development of an analytical scaling for the SOL width, which has compared positively with experimental data [15]. A mismatch between the measured Alcator C-Mod SOL width and the results of our analytical scaling has prompted this detailed comparison between simulations and experiments, that we present below.

\section{GBS modeling of the C-Mod SOL and comparison with experiment}

Four simulations of C-Mod SOL dynamics were carried out using the physical and dimensionless parameters given in table 1 . We assume $\bar{R}=0.67 \mathrm{~m}, \bar{a}=0.216 \mathrm{~m}, q=2.7, \hat{s}=1.3$
$, \kappa=1, m_{i}=2 m_{p}, m_{i} / m_{e}=1600$, and $\tau=2$ at the LCFS. (Note that $\tau$ is involved only in the definition of $\rho_{s}$ in these simulations, since there are no $T_{i}$ fluctuations.) The factor $\tau=2$ is obtained from typical charge exchange recombination spectroscopy (CXRS) measurements of the Alcator C-Mod edge [25]. The source terms $S_{n} \approx 1.5-1.8$ and $S_{T_{e}} \approx 1.5-1.6$ were adjusted to obtain a good match between temperature and density at the LCFS of the simulations and the reference $\bar{n}$ and $\bar{T}_{e}$, which were obtained from Langmuir probe measurements. The volume integral of the $T_{e}$ source yields roughly $1 \mathrm{MW}$, which is consistent with the C-Mod SOL power in the $3.8 \mathrm{~T}$ cases and about twice that found experimentally in the $2.8 \mathrm{~T}$ cases. As the sources were adjusted to match $\bar{n}$ and $\bar{T}_{e}$, the plasma profiles appear to be rather insensitive to the injected power, and the statistical moments were not affected.

The GBS simulations evolve a 3D volume within a computational grid of size $\left(n_{\theta}, n_{r}, n_{\varphi}\right)=(1384,128,256)$. These dimensions allow a resolution of 128 toroidal modes with $k_{\theta, \max } \rho_{s} \approx 1-1.3 \sim 3 \mathrm{~mm}^{-1}$. The radial domain of the simulation is $100 \rho_{s}$, with the sources centered at $r_{0}=20 \rho_{s}$ and an additional buffer region used in the last $10 \rho_{s}$. Altogether, the usable radial extent is $70 \rho_{s}$, which gives a $2-3 \mathrm{~cm}$ SOL length depending on the simulated case.

The radial boundary conditions used are Neumann for $n, \omega, v_{\| i}, v_{\| e}$, and $T_{e}$, while the electrostatic potential has a boundary condition $\partial_{r} \phi=0$ at the inner boundary and $\phi=3\left\langle T_{e}\right\rangle_{t}$ at the outer boundary. The meaningful physical inner boundary of the simulation is in fact the location of the source, $r_{0}$, where the time averaged temperature and density are $\left\langle T_{e}\right\rangle_{t} \approx 1$ and $\langle n\rangle_{t} \approx 1$. We interpret $r_{0}$ in our simulations as being the LCFS. Near the source injection point the potential satisfies $\langle\phi\rangle_{t} \approx 3\left\langle T_{e}\right\rangle_{t}$ and $\left\langle\partial_{r} \phi\right\rangle_{t}=0$ through coupling with the temperature profile [26] (i.e. the temperature peaks at the source, giving $\left\langle E_{r}\right\rangle_{t} \approx 0$ ). This computational setup is compatible with Langmuir probe measurements of the SOL profiles across the LCFS, while at the same time it does not constrain the amplitude of the fluctuations or the instantaneous value of $E_{r}$.

\subsection{Time averaged profiles}

GBS simulations yield a reasonably good match to the C-Mod profiles as measured using the reciprocating Langmuir probe. Both simulation and experiment show a relatively flat far SOL and a steeper near SOL. We have fit the GBS profiles using a function of the form $p=c_{1} \exp \left(-\left(r-r_{0}\right) / L_{p}\right.$ $\left.{ }_{, 1}\right)+c_{2} \exp \left(-\left(r-r_{0}\right) / L_{p, 2}\right)$, which gives $L_{p, 1} \approx 15 \bar{\rho}_{s} \approx 6 \mathrm{~mm}$ and $L_{p, 1} \approx 70 \bar{\rho}_{s} \approx 2 \mathrm{~cm}$. It is still possible to fit the GBS profiles using one single scale length, although the agreement between GBS and the fit decreases significantly. The other simulated cases yield similar profiles. It appears that profile formation involves physical processes not included in our analytical scaling [15]. The analysis of the background profiles will be addressed in the future using the so-called 'mirror' Langmuir probe [27], which has the accuracy needed to unequivocally resolve the separate SOL scale lengths. 

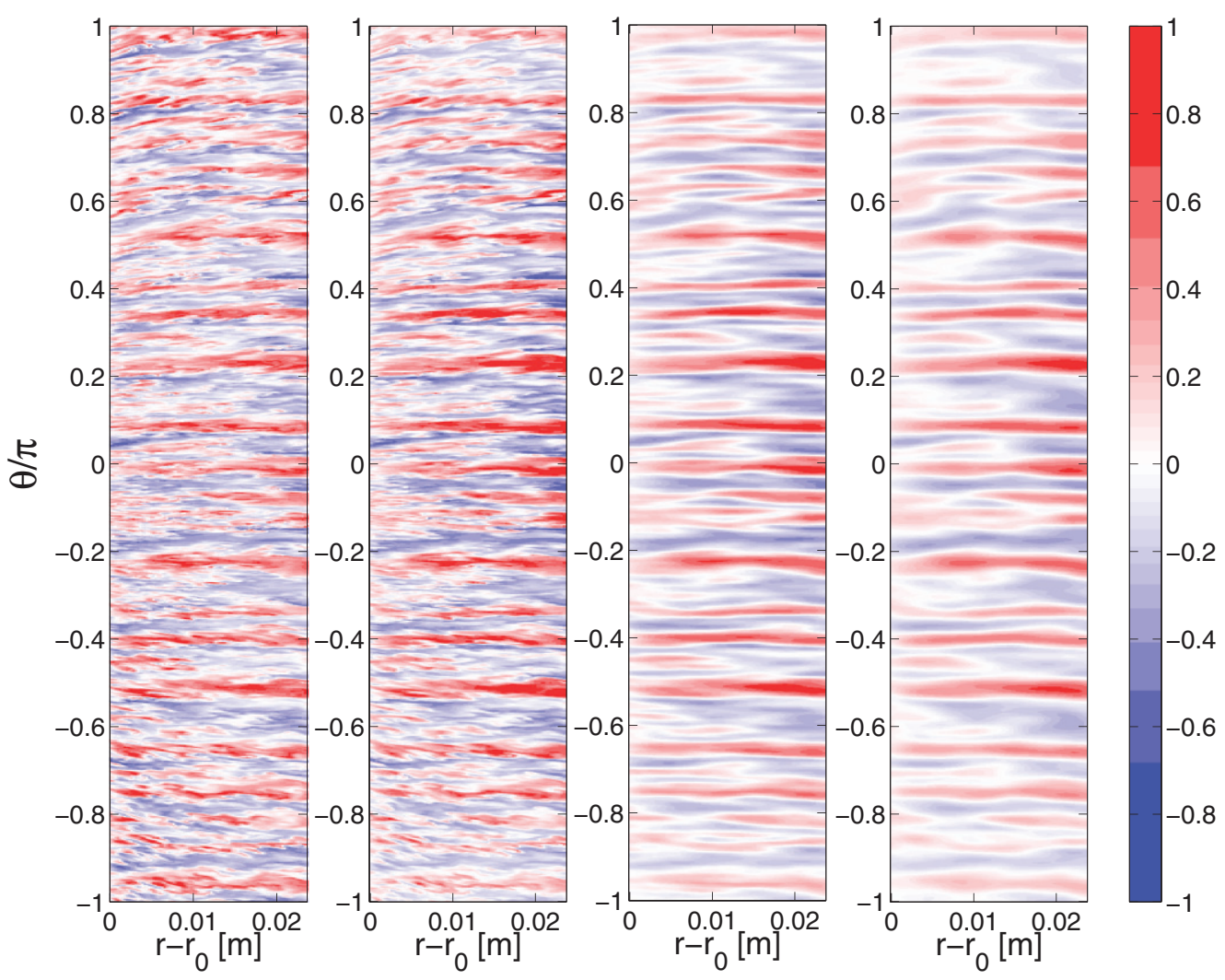

Figure 1. Poloidal cross section of the simulated C-Mod SOL show the effects of GPI finite resolution and tilt respect to the magnetic field lines on the turbulent fluctuations. Left to right: $\delta n / n, \delta D_{\alpha} / D_{\alpha}$ (no smoothing), $\delta D_{\alpha} / D_{\alpha}$ with smoothing due to GPI resolution, and $\delta D_{\alpha} / D_{\alpha}$ with full smoothing due to GPI resolution and magnetic field line tilt.

\subsection{Evaluation of $\delta D_{\alpha} / D_{\alpha}$ and effects of smoothing}

In order to compare the GBS results against GPI measurements, the GBS density and temperature is used to calculate the perturbations in $D_{\alpha}$ light emission. This is achieved through a local parametrization of the emissivity using $D_{\alpha} \propto n^{\alpha} T_{e}^{\beta}$, with the exponents $\alpha\left(n, T_{e}\right)$ and $\beta\left(n, T_{e}\right)$ computed from a local fit to the emission profiles calculated with DEGAS-2 [18]. Assuming small excursions in emissivity with respect to the background values, we compute the fluctuating part of $D_{\alpha}$,

$$
\frac{\delta D_{\alpha}}{D_{\alpha}} \approx \alpha \frac{\delta n}{n}+\beta \frac{\delta T_{e}}{T_{e}}
$$

with the exponents $\alpha$ and $\beta$ obtained using the toroidally averaged temperature and density $\left\langle T_{e}\right\rangle_{\varphi}$ and $\langle n\rangle_{\varphi}$.

In order to match the GPI space and time resolution, and the magnetic field line tilt of $8^{\circ}$ respect to the toroidal angle, the GBS results are smoothed in time and space. The finite resolution is simulated with a simple moving average filter over a $3 \times 3 \mathrm{~mm}$ extension, while the finite bandwidth of the digitized signal $f=0.4 \mathrm{MHz}$ is simulated by averaging the simulation data over $2.5 \mu \mathrm{s}$. An additional poloidal smoothing over $8 \mathrm{~mm}$ is used to take into account the field line tilt due to the safety factor. The GBS turbulent properties are calculated using 2D data output with a sampling frequency of about $5 \mathrm{MHz}-1000$ time snapshots spanning $\sim 1 \mathrm{~ms}$ are averaged for each simulated case.
The influence of this time and spatial smoothing is demonstrated in figure 1. From left to right, we display density fluctuations $\delta n / n=\left(n-\langle n\rangle_{\varphi}\right) /\langle n\rangle_{\varphi}, \delta D_{\alpha} / D_{\alpha}$ without smoothing, $\delta D_{\alpha} / D_{\alpha}$ with smoothing due to photodiode time and spatial resolution $(3 \times 3 \mathrm{~mm}+2.5 \mu \mathrm{s})$, and $\delta D_{\alpha} / D_{\alpha}$ with full smoothing, i.e. diode plus $8 \mathrm{~mm}$ vertical smoothing from the field line tilt. Pannels 2 and 3 are smoothed in time, but the time averaging window is short enough to give the appearance of an instantaneous snapshot. The $\delta D_{\alpha} / D_{\alpha}$ fluctuations are in fact very similar to the density fluctuations up to a small enhancement due to adding the temperature fluctuations and the values of $\alpha$ and $\beta$. The $T_{e}$ fluctuations are very well correlated with the $n$ fluctuations and have a similar magnitude. However, as smoothing is applied, the fluctuation level is progressively decreased, in particular, at small spatial scales. Note that the poloidal extension of the plots is $2 \pi a \approx 1.25 \mathrm{~m}$. From this figure, it can be estimates that the typical turbulent structures have radial and poloidal lengths of the order of a cm.

The effects of smoothing can be observed, additionally, in the poloidal wave number $\left(k_{\mathrm{pol}}\right)$ spectrum of the fluctuations. The successive application of smoothing results in a sharp cut-off at the wave number that corresponds to the poloidal spatial filter width, $k_{\text {pol }} \approx 125 \mathrm{~m}^{-1}$. As a result, the slope of the power law decay of the spectral amplitude is substantially increased-this phenomenon was also observed in the previous GEMR simulations [9]. The correlation lengths are also increased by the smoothing, while the correlation time, on the 

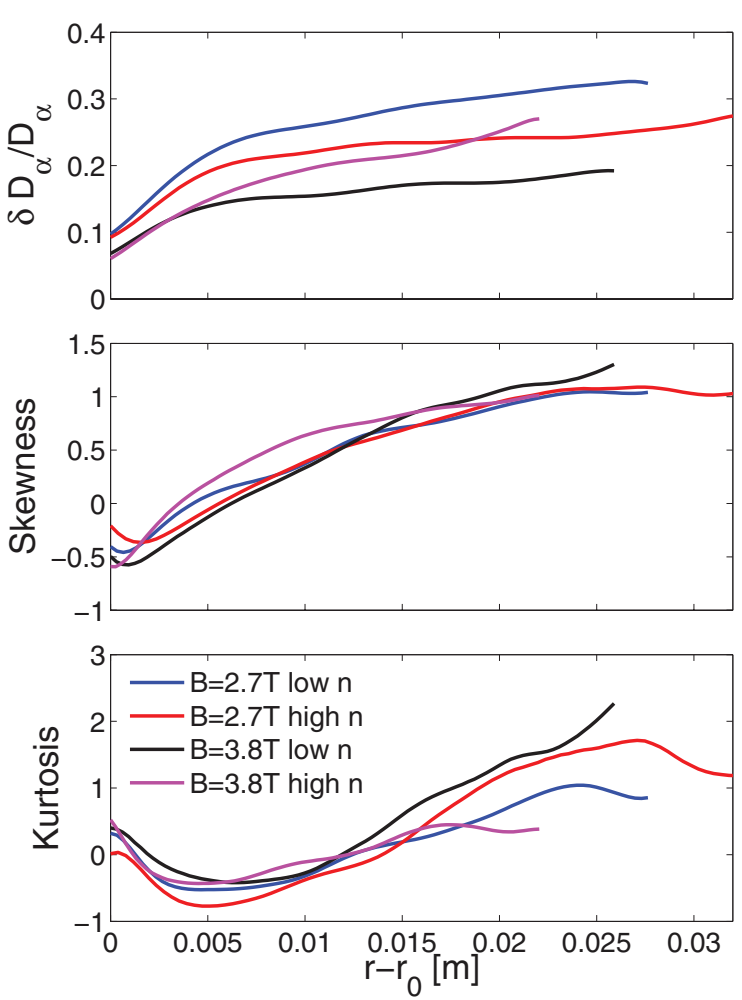
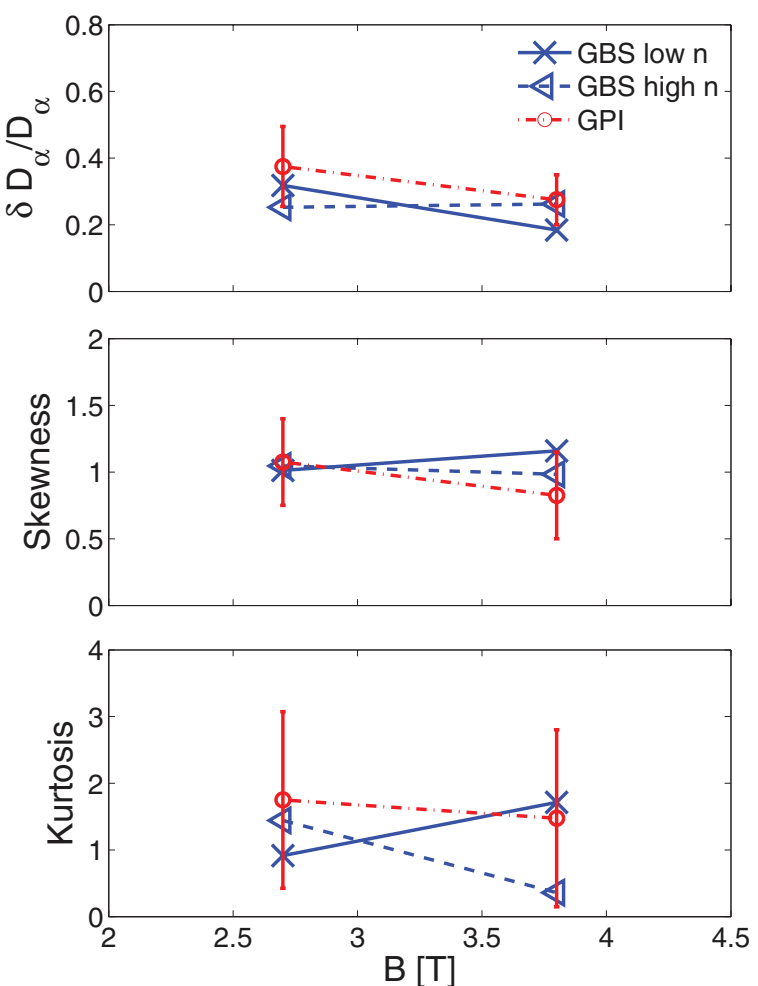

Figure 2. Left: Statistical moments of $\delta D_{\alpha} / D_{\alpha}$ in GBS simulations for cases with $B=2.7 \mathrm{~T}$ and $3.8 \mathrm{~T}$, low and high density cases. Right: Comparison of $\delta D_{\alpha} / D_{\alpha}$ statistical moments obtained from GBS data with the results published in [9]. The comparison is carried out using GBS data from $r-r_{0}>2 \mathrm{~cm}$, where the simulated moment profiles become relatively flat.

other hand, is not affected as much. From here onwards, we discuss the results obtained from the simulated $\delta D_{\alpha} / D_{\alpha}$ with the all the smoothing applied.

\subsection{Statistical moments of $\delta D_{\alpha} / D_{\alpha}$}

We are interested in studying quantities such as the root-meansquare (RMS) fluctuation level, as well as the skewness, and the kurtosis of the probability distribution function (PDF) of the fluctuations. These, in turn, allow us to quantify the intensity of the fluctuations and their nature, in particular, the presence of large amplitude intermittent transport events (blobs). Using 3D data output from GBS, we have reconstructed the $\delta D_{\alpha} / D_{\alpha} \mathrm{PDF}$ and extracted its moments as a function of radius, as shown in on the left pannel of figure 2. GBS simulations show radially increasing fluctuation level, skewness, and kurtosis, with the fluctuations being weakest and more Gaussian near the LCFS. The far SOL appears to be dominated by transient transport events with large amplitude.

A comparison between GBS and Alcator C-Mod data is shown on the right pannel of figure 2. Here, we plot the moments obtained from GBS, averaged for $r-r_{0}>2 \mathrm{~cm}$, where the radial profiles of the moments are relatively flat. Superimposed are the measurements published in [9], demonstrating good agreement between simulations and experiment. The relative fluctuation levels, skewness, and kurtosis calculated in GBS appear to reproduce experimental measurements essentially within the error bars. (Here, the error bars reflect shot to shot variation, and they are obtained by grouping all the discharges with the same $B$ ). We note that the fluctuation level $\delta D_{\alpha} / D_{\alpha} \sim 0.3$, the skewness $\sim 1$, and the kurtosis $\sim 2$ are similar to the values found in the past in $2 \mathrm{D}$ flux driven simulations using the TOKAM2D, ESEL, and SOLT codes $[3,5,6,28]$.

\subsection{Spatial and temporal characterization of the C-Mod SOL turbulent structures and Their propagation}

The turbulence is characterized by analyzing time traces of neighboring sensors separated by a radial or poloidal distance $\delta$ (of a few mm's), determined by the spacing of the GPI sensors. The quantities of interest are the radial and poloidal correlation lengths $L_{\mathrm{rad}}$ and $L_{\mathrm{pol}}$; the auto correlation time $\tau_{\text {auto }}$; and the radial and poloidal turbulent velocities $v_{\text {rad }}$ and $v_{\text {pol }}$. They are computed following the same procedure as in [9] and they are defined as follows:

$$
\begin{gathered}
L=1.66 \frac{\delta}{\sqrt{-\ln C_{i j}}} \\
C_{i i}\left(\tau_{\text {auto }}\right)=\frac{1}{2} \\
v=\frac{\delta}{\left\langle\tau_{v}\right\rangle}
\end{gathered}
$$

where $C_{i j}$ is the zero-time-delay cross correlation function between two neighboring sensors. The velocity involves $\tau_{v}$, which is the time delay that maximizes the cross-correlation between two neighboring sensors. Note here that the auto 

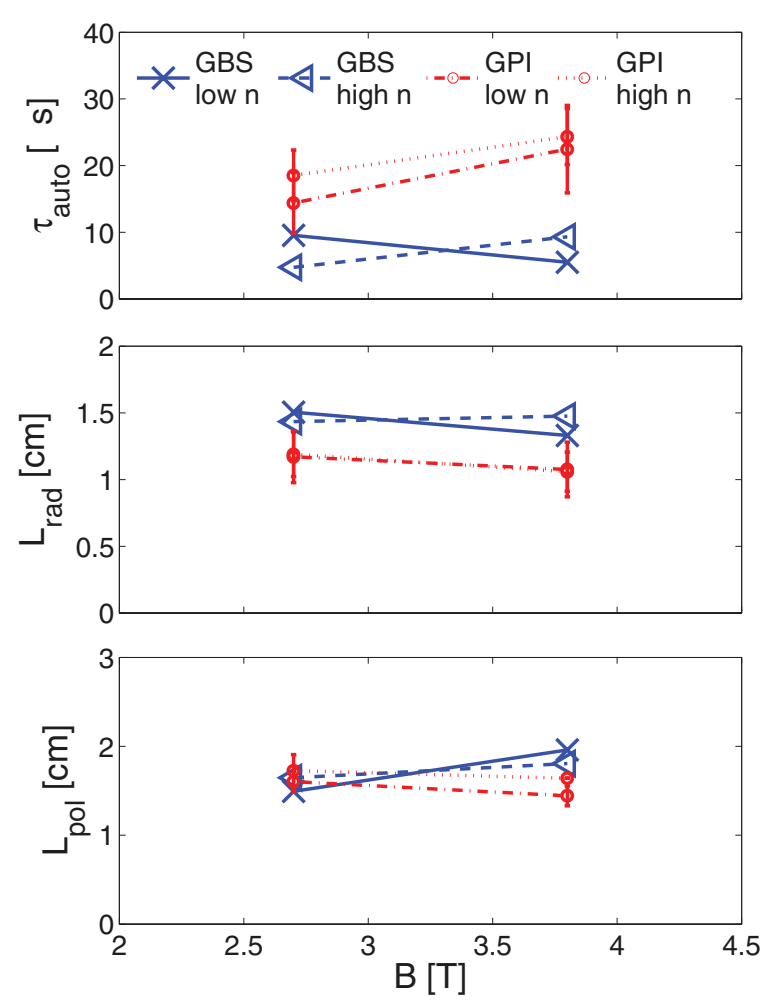

Figure 3. Comparison of the autocorrelation time $\tau_{\text {auto }}$, and radial and poloidal correlation lengths $L_{\mathrm{rad}}$ and $L_{\mathrm{pol}}$ (top, center, and bottom, respectively).

correlation time is defined using the half-width of the halfmaximum of the correlation function, while the correlation length involves the full-width of the half-maximum.

In order to obtain improved statistics, we consider the entire low-field-side region at $r-r_{0}>2 \mathrm{~cm}$, and the turbulent properties are computed as a radial and poloidal average. It was checked that there is little radial variation in the simulated correlations.

First, we examine the auto correlation times, which are displayed on the top pannel of figure 3. The GBS simulations underestimate the correlation times significantly for all the cases studied. This phenomenon was also observed in GEMR simulations and it remains unexplained. The radial and poloidal correlation lengths, on the other hand, show good agreement, with $L_{\mathrm{rad}} \sim L_{\mathrm{pol}} \approx 1.5 \mathrm{~cm}$ in both experiment and simulations, regardless of magnetic field or density. The mode width is a very robust feature within GBS simulations, and changes little at experimentally relevant parameters. The radial correlation lengths obtained in GBS are slightly overestimated, but near the error bars indicating shot to shot variations.

The radial and poloidal turbulent propagation velocities are shown in figure 4 . The radial speeds are close to $1 \mathrm{~km} \mathrm{~s}^{-1}$, which is roughly consistent with blob propagation speedsthese speeds can vary within an order of magnitude depending on the experiment. Since blobs are propelled by turbulencedriven dipole electric fields, the magnitude of $v_{\text {rad }}$ is in fact indicating that the poloidal mode structure is correctly captured. The poloidal velocity, on the other hand is overestimated in 3 out of 4 cases. The synthetic GPI $v_{\text {pol }}$ profiles are essentially flat in the far SOL. A possible cause for the disagreement could be the steepness of the $\phi$ profile, which is closely coupled to $T_{e}$ through the electron adiabaticity condition and sheath physics [26].

As a check, we have computed the blob velocity expected from standard blob theory (e.g. [29]), using $L_{\text {rad }}$ as the blob size. This implies the assumption that the turbulent structures detected are indeed blobs, which cannot be guaranteed without pattern recognition software. The reference blob size is $L_{*}=\left(4 L_{c}^{2} /\left(\bar{\rho}_{\mathrm{s}} \bar{R}\right)\right)^{1 / 5} \bar{\rho}_{\mathrm{s}} \approx 7 \mathrm{~mm}$, while the reference blob velocity is $v_{*}=\left(2 L_{c} \bar{\rho}_{s}^{2} / \bar{R}^{3}\right) \bar{c}_{s} \approx 6-8 \mathrm{~km} \mathrm{~s}^{-1}$ ( $L_{c}=2 \pi q \bar{R}$ is the connection length). Therefore, blobs have $L_{\mathrm{rad}} / L_{*} \approx 2$, i.e. they are in the sheath-dissipative regime where parallel currents damp radial propagation. The blob velocity expected from the analytical theory is $v \sim v_{*} \sqrt{L_{\mathrm{rad}} / L_{*}} /\left(1+\sqrt{2}\left(L_{\mathrm{rad}} / L_{*}\right)^{5 / 2}\right) \approx 0.7-1.6 \mathrm{~km} \mathrm{~s} \mathrm{~s}^{-1}$, which is in good agreement with $v_{\text {rad }}$ as shown in figure 4 .

\subsection{Wave number and frequency spectra of the fluctuations}

The fluctuation power spectrum as a function of the poloidal wave number for two cases $(B=2.7 \mathrm{~T}$, low $n$ and $B=3.8 \mathrm{~T}$, high $n$ ) are shown on the top panels of figure 5. The fluctuation levels have been re-scaled at the lowest $k_{\text {pol }}$ available for the GPI data. Both spectra are obtained from Fourier transforms. Good agreement is found between the spectra at low wave numbers, where the fluctuations are most intense. Both experiment and simulations show a peak in the fluctuations at roughly $k_{\text {pol }} \approx 100 \mathrm{~m}^{-1}$, which matches the poloidal correlation length $L_{\mathrm{pol}} \approx 1 \mathrm{~cm}$. In normalized units, this corresponds to roughly $10 \rho_{s}$, which is the typical dominant $k_{\text {pol }}$ found for a wide range of parameters. As noted before, there is a sharp cut-off in the GBS $\delta D_{\alpha} / D_{\alpha}$ spatially smoothed spectrum at around $k_{\mathrm{pol}} \approx 125 \mathrm{~m}^{-1}$ due to the $8 \mathrm{~mm}$ vertical smoothing applied to simulated the field line tilt. The unsmoothed data shows a similar power law decay slope at high $k$ compared to the C-Mod GPI. It is possible that the spatial smoothing applied to the synthetic GPI data is excessive.

The fluctuation power as a function of the frequency is shown just below, on the bottom pannels of figure 5, for the same cases. The fluctuation levels are renormalized at the lowest frequency available for the GBS simulations $(\sim 1 \mathrm{kHz})$ which is given by the total length of the simulations. Spectra for modes with positive and negative propagation velocities as measured by the GPI are shown. For the GBS data, the $+k$ and $-k$ spectra refer to the positive and negative frequencies obtained from the Fourier transform. The most salient feature here is the power law decay of the fluctuation power with the frequency, which is very well described by the GBS simulations.

Finally, it is noted that there is overall little variation in both $k_{\mathrm{pol}}$ and $f$ spectra across the radius in the GBS simulations. For this comparison we have taken a radial average around $2 \pm 0.2 \mathrm{~cm}$.

\section{Summary and conclusions}

We have carried out a quantitative comparison between GBS, a drift-Braginskii turbulence code, and GPI turbulence 

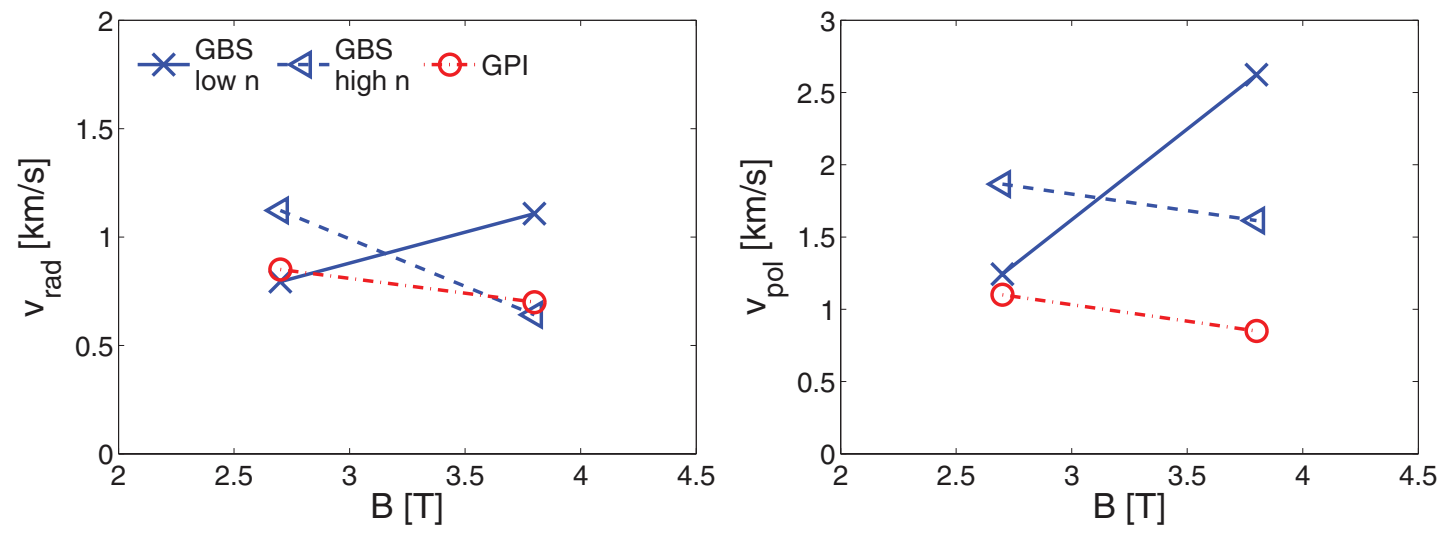

Figure 4. Comparison of the turbulent propagation velocities $v_{\text {rad }}$ (left) and $v_{\text {pol }}$ obtained from the time delay that maximizes the cross correlation function between two neighboring sensors.
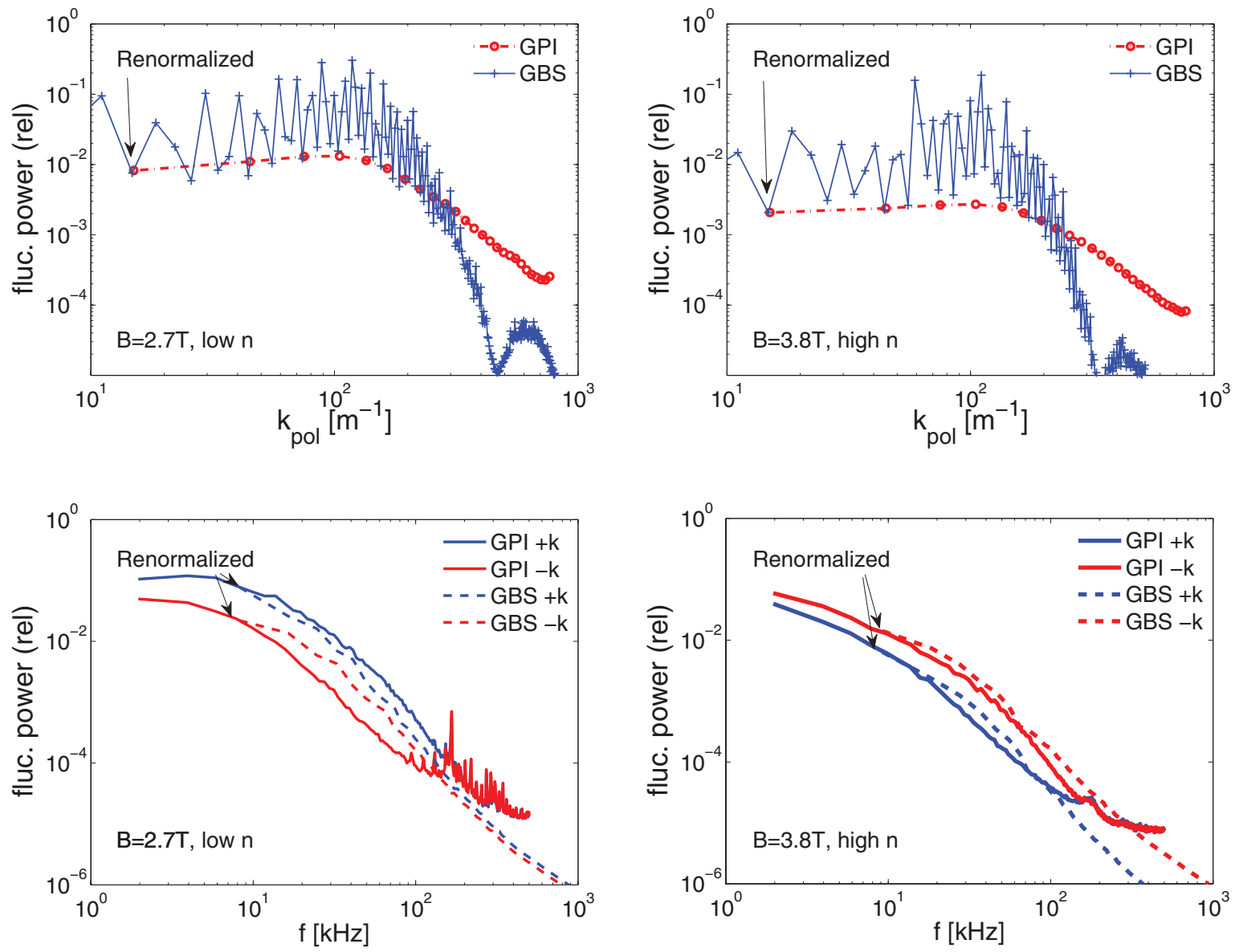

Figure 5. Comparison of the wavenumber (top) and frequency (bottom) spectra for the $B=2.7 \mathrm{~T}$ low $n$ case (left) and for the $B=3.8 \mathrm{~T}$ high $n$ case (right). The power spectra are renormalized where indicated by the arrows. The features at around $200 \mathrm{kHz}$ are due to detector pickup and not features of the plasma.

measurements in the Alcator C-Mod SOL. In contrast to our previous simulation work [12-15], which concentrated on building a predictive theory of the SOL width, this validation exercise provides a more comprehensive view of SOL turbulent processes. The comparison results show good agreement in almost all the observables considered, indicating that far SOL turbulence is well described in GBS simulations. This outcome further increases our confidence in the use of 3D flux-driven codes to model SOL turbulent dynamics.

The quantities analyzed in the comparison are statistical moments of the PDF; the correlation lengths, auto correlation time, and propagation velocities; and the wave number and frequency spectral powers. The comparison was carried out by converting GBS primitive variables, $n$ and $T_{e}$, into $D_{\alpha}$ fluctuations with a simple parametrization of the emissivity. 
Generally, the fluctuating component $\delta D_{\alpha} / D_{\alpha}$ is quite similar to $\delta p / p$. Poloidal, radial, and time smoothing are then applied on the synthetic $\delta D_{\alpha} / D_{\alpha}$ data in order to simulate the finite spatial and temporal resolution of the GPI diagnostic.

First, GBS simulations resulted in reasonable time averaged profiles, which in fact enables a meaningful comparison. Very good agreement is found for the simulated statistical moments, which are within the experimental error bars representing shot to shot variation. The main quantitative difference between these simulations and a previous publication [9] appears to be a normalized $D_{\alpha}$ fluctuation level of roughly $25-35 \%$ with GBS compared to 5-15\% with GEMR.

The simulated correlation lengths and the propagation velocities are also in reasonable agreement with experiment, yielding $L \sim 1 \mathrm{~cm}$ and $v \sim 1 \mathrm{~km} \mathrm{~s}^{-1}$, except for $v_{\text {pol }}$ which was significantly overestimated by GBS. The auto correlation times, on the other hand, are underestimated in the GBS simulations by a factor of 2 . The wave number and frequency spectra also show good agreement, in particular, the $k_{\text {pol }}$ spectra peak at $k_{\mathrm{pol}} \approx 1 \mathrm{~cm}^{-1}$ and they have the same shape below the cut off due to GPI resolution smoothing. The frequency spectra show very similar power law decay from $f=10 \mathrm{kHz}$ onwards.

Given the strongly skewed PDF found in the far SOL of the GBS simulations, it appears that a sizable fraction of the radial transport found is due to intermittent events. Indeed, as a check, we have computed the expected blob velocity stemming from blobs with size $L_{\text {rad }}$. The result is compatible with the radial velocities extracted from the two point correlations. Additionally, it must be mentioned that there is altogether little case-to-case variation in the profiles or the PDFs. Altogether, the simulation results and the experimental measurements are consistent with the paradigms of universality and self-organization for SOL turbulence (e.g. [30]).

Finally, we stress that this initial validation exercise will be expanded, on one hand, by using a more advanced model including ion temperature dynamics and plasma shaping [20, 31 , and on the other hand, by comparing GBS against new experimental data from C-Mod's 2014 campaign. The new experimental series will include other advanced diagnostics, such as the mirror Langmuir probe and CXRS-GPI measurements of $T_{i}$. A three-way comparison between mirror probe, GPI, and GBS data will allow for a more exhaustive quantification of the turbulent dynamics and profile formation in the near and far SOL.

\section{Acknowledgments}

The authors would like to thank S Jolliet, J Loizu, A Mosetto, F Riva, C Wersal and T-M Tran for useful discussions. Parts of the simulations presented herein were carried out at the Swiss National Supercomputer Center (CSCS) under project ID s346; parts were carried out at the National Energy Research Scientific Computing Center (NERSC) under project GBSSOL; and parts were carried out using the HELIOS supercomputer system at the Computational Simulation Centre of the International Fusion Energy Research Centre (IFERCCSC), Aomori, Japan, under the Broader Approach collaboration between Euratom and Japan, implemented by Fusion for Energy and JAEA. This work was carried out within the framework of the EUROfusion Consortium. It was supported in part by the Swiss National Science Foundation, in part by USDoE awards DE-FC02-99ER54512 and DE-AC0209CH11466, and received funding from the Euratom research and training programme 2014-2018 under grant agreement number 633053 . The views and opinions expressed herein do not necessarily reflect those of the European Commission.

\section{References}

[1] Zweben S J, Boedo J A, Grulke O, Hidalgo C, LaBombard B, Maqueda R J, Scarin P and Terry J L 2007 Edge turbulence measurements in toroidal fusion devices Plasma Phys. Control. Fusion $49 \mathrm{~S} 1$

[2] Krasheninnikov S I 2001 On scrape off layer plasma transport Phys. Lett. A 283 368-70

[3] Sarazin Y et al 2003 Theoretical understanding of turbulent transport in the SOL J. Nucl. Mater. 313 796-803

[4] Garcia O E, Horacek J, Pitts R A, Nielsen A H, Fundamenski W, Naulin V and Rasmussen J J 2007 Fluctuations and transport in the TCV scrape-off layer $\mathrm{Nucl}$. Fusion 47667

[5] Myra J R et al 2011 Reduced model simulations of the scrapeoff-layer heat-flux width and comparison with experiment Phys. Plasmas 18012305

[6] Russell D A, Myra J R, D’Ippolito D A, Munsat T L, Sechrest Y, Maqueda R J, Stotler D P, Zweben S J and The NSTX Team 2011 Comparison of scrape-off layer turbulence simulations with experiments using a synthetic gas puff imaging diagnostic Phys. Plasmas $\mathbf{1 8} 022306$

[7] Scott B D 2005 Drift wave versus interchange turbulence in tokamak geometry: linear versus nonlinear mode structure Phys. Plasmas 12062314

[8] Ribeiro T T and Scott B 2008 Gyrofluid turbulence studies of the effect of the poloidal position of an axisymmetric debye sheath Plasma Phys. Control. Fusion 50055007

[9] Zweben S J, Scott B D, Terry J L, LaBombard B, Hughes J W and Stotler D P 2009 Comparison of scrape-off layer turbulence in Alcator C-Mod with three dimensional gyrofluid computations Phys. Plasmas 16082505

[10] Ricci P, Halpern F D, Jolliet S, Loizu J, Mosetto A, Fasoli A, Furno I and Theiler C 2012 Simulation of plasma turbulence in scrape-off layer conditions: the GBS code, simulation results and code validation Plasma Phys. Control. Fusion 54124047

[11] Zeiler A, Drake J F and Rogers B 1997 Nonlinear reduced Braginskii equations with ion thermal dynamics in toroidal plasma Phys. Plasmas 4 2134-8

[12] Ricci P and Rogers B N 2013 Plasma turbulence in the scrape-off layer of tokamak devices Phys. Plasmas 20010702

[13] Mosetto A, Halpern F D, Jolliet S, Loizu J and Ricci P 2013 Turbulent regimes in the tokamak scrape-off layer Phys. Plasmas 20092308

[14] Halpern F D, Ricci P, Jolliet S, Loizu J and Mosetto A 2014 Theory of the scrape-off layer width in inner-wall limited tokamak plasmas Nucl. Fusion 54043003

[15] Halpern F D et al 2013 Theory-based scaling of the SOL width in circular limited tokamak plasmas Nucl. Fusion 53122001

[16] Scott S et al 2007 Overview of the Alcator C-Mod research programme Nucl. Fusion 47 S598

[17] Terry J L et al 2003 Observations of the turbulence in the scrape-off-layer of Alcator C-Mod and comparisons with simulation Phys. Plasmas 10 1739-47 
[18] Stotler D P, Boedo J, LeBlanc B, Maqueda R J and Zweben S J 2007 Progress towards the validation of models of the behavior of neutral helium in gas puff imaging experiments J. Nucl. Mater. 363-5 686-92 (Plasma-Surface Interactions-17)

[19] Braginskii S I 1965 Transport processes in a plasma Rev. Plasma Phys. 1205

[20] Mosetto A, Halpern F D, Jolliet S, Loizu J and Ricci P 2015 Finite ion temperature effects in SOL turbulence Phys. Plasmas 22012308

[21] Loizu J, Ricci P, Halpern F D and Jolliet S 2012 Boundary conditions for plasma fluid models at the magnetic presheath entrance Phys. Plasmas 19122307

[22] Scott B 2001 Shifted metric procedure for flux tube treatments of toroidal geometry: avoiding grid deformation Phys. Plasmas 8 447-58

[23] Scott B D 2005 Free-energy conservation in local gyrofluid models Phys. Plasmas 12102307

[24] Halpern F D, Jolliet S, Loizu J, Mosetto A and Ricci P 2013 Ideal ballooning modes in the tokamak scrape-off layer Phys. Plasmas 20052306

[25] Churchill R M, Theiler C, Lipschultz B, Dux R, Pütterich T, Viezzer E, Alcator C-Mod Team and ASDEX Upgrade Team 2013 Development of the gas puff charge exchange recombination spectroscopy (GP-CXRS) technique for ion measurements in the plasma edge Rev. Sci. Instrum. 84093505

[26] Loizu J, Ricci P, Halpern F D, Jolliet S and Mosetto A 2013 On the electrostatic potential in the scrape-off layer of magnetic confinement devices Plasma Phys. Control. Fusion 55124019

[27] LaBombard B, Golfinopolous T, Terry J L, Brunner D, Davis E, Greenwald M, Hughes J W and Alcator C-Mod Team 2014 Phys. Plasmas 21056108

[28] Garcia O E, Horacek J, Pitts R A, Nielsen A H, Fundamenski W, Graves J P, Naulin V and Rasmussen J J 2006 Interchange turbulence in the TCV scrape-off layer Plasma Phys. Control. Fusion $48 \mathrm{~L} 1$

[29] D'Ippolito D A, Myra J R and Zweben S J 2011 Convective transport by intermittent blob-filaments: comparison of theory and experiment Phys. Plasmas $\mathbf{1 8} 060501$

[30] van Milligen B Ph, Sánchez R, Carreras B A, Lynch V E, LaBombard B, Pedrosa M A, Hidalgo C, Gonçalves B, Balbín R and The W7-AS Team 2005 Additional evidence for the universality of the probability distribution of turbulent fluctuations and fluxes in the scrape-off layer region of fusion plasmas Phys. Plasmas $\mathbf{1 2} 052507$

[31] Jolliet S, Halpern F D, Loizu J, Mosetto A and Ricci P 2014 Aspect ratio effects on limited scrape-off layer plasma turbulence Phys. Plasmas 21022303 